\title{
CROSSOVER BETWEEN THE DENSE ELECTRON-HOLE PHASE AND THE BCS EXCITONIC PHASE IN QUANTUM DOTS
}

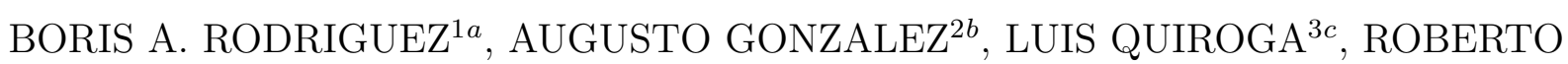 \\ CAPOTE4 ${ }^{4}$, and FERNEY J. RODRIGUEZ ${ }^{3]}$ \\ ${ }^{1}$ Departamento de Fisica, Universidad de Antioquia, AA 1226, Medellin, Colombia \\ ${ }^{2}$ Instituto de Cibernetica, Matematica y Fisica Calle E 309, Vedado, Habana 4, Cuba \\ ${ }^{3}$ Departamento de Fisica, Universidad de los Andes, AA 4976, Bogota, Colombia \\ ${ }^{4}$ Centro de Estudios Aplicados al Desarrollo Nuclear, Calle 30 No 502, Miramar, La Habana, \\ Cuba
}

(October 1, 2018)

\begin{abstract}
Second order perturbation theory and a Lipkin-Nogami scheme combined with an exact Monte Carlo projection after variation are applied to compute the ground-state energy of $6 \leq N \leq 210$ electron-hole pairs confined in a parabolic two-dimensional quantum dot. The energy shows nice scaling properties as $N$ or the confinement strength is varied. A crossover from the high-density electron-hole phase to the BCS excitonic phase is found at a density which is roughly four times the close-packing density of excitons.
\end{abstract}




\section{INTRODUCTION}

As we understand, the interest in electron-hole states (excitons) in semiconductor physics is motivated by two facts. First, excitons have a bosonic character (as they are made up of a pair of fermions) and, thus, the many-exciton system is a candidate for a Bose condensate. This possibility was envisaged long ago [1], but regained attention in the last years after the Bose condensation of alcali atoms was achieved [2]. Experimentally, signals of Bose-Einstein statistics have been identified in the photoluminiscence of quantum wells under strong laser pumping [3], and indirect excitons in quantum wells are being manipulated via applied stress and inhomogeneous electric fields [4] to reach the densities needed for Bose condensation. On the other hand, excitons are at the basis of many optical properties of semiconductors [5]. Recent experimental works have focused on the lowest dimensional structures, and very interesting properties have been found in the photoluminiscence of quantum wires [6] and single quantum dots [7].

In the present paper, we study a two-dimensional quantum dot with a number of electronhole pairs, $6 \leq N \leq 210$, i. e. intermediate between the very small dot [7] and the quantum well [3]. We study the dot at strong and intermediate confinement regimes by means of second-order perturbation theory and a variational (BCS) procedure. The main results of the paper may be summarised as follows.

We found a breakdown of perturbation theory and a significant BCS pairing roughly at the same confinement strength, corresponding approximately to four times the closepacking density of excitons (or four times the Mott transition density). We notice that the BCS calculations were performed within the Lipkin-Nogami scheme [8] with exact projection onto the $N$-pair sector [9] to avoid the incorrect behaviour of the naive BCS function in a finite system [10]. Second, we found that the energy depends on $N$ and the confinement strength in a scaled way.

Our paper is complementary to [11, 12], in which the multiexcitonic quantum dot is also studied. In [1], we found that the far-infrared absorption of the dot is dominated by a giant- 
dipole resonance similar to the collective state apearing in nuclei [13] and metallic clusters [14]. In paper [12], the Bethe-Goldstone equations (the independent-pair approximation in Nuclear Physics [15]) are applied to study small $(2 \leq N \leq 6)$ clusters. The frequency for optical absorption with creation of an electron-hole pair shows a very interesting behaviour related to the apparent instability of the free (not confined) four-exciton cluster in two dimensions.

\section{PERTURBATION THEORY}

We study a direct-band-gap semiconductor with two parabolic bands. $N$ electrons and $N$ holes are created by, e. g., strong laser pumping. We shall ignore recombination processes and electron-hole exchange. A model like this have been employed for the analysis of collective excitations in bulk semiconductors [16]. The particles are forced to move in a two-dimensional region confined by a parabolic potential. This is a common approach in the study of self-assembled quantum dots [17]. For simplicity, we take $m_{h}=m_{e}$ and the same confining potential for both particles. Up to 210 electron-hole pairs will be allowed in the dot.

The Hamiltonian of the system in oscillator units is written as

$$
\frac{H}{\hbar \omega}=\frac{1}{2} \sum_{\alpha=1}^{2 N}\left(\vec{p}_{\alpha}^{2}+\vec{r}_{\alpha}^{2}\right)+\beta \sum_{\alpha<\gamma} \frac{q_{\alpha} q_{\gamma}}{\left|\vec{r}_{\alpha}-\vec{r}_{\gamma}\right|}
$$

where $\omega$ is the dot frequency, $q_{\alpha}=-1$ for electrons and +1 for holes. This Hamiltonian

depends only on one constant, $\beta=\sqrt{\left(\frac{m e^{4}}{\kappa^{2} \hbar^{2}}\right) /(\hbar \omega)}=\sqrt{E_{c} /(\hbar \omega)}$, where $E_{c}$ is the Coulomb characteristic energy. $m$ is the electron effective mass, and $\kappa$ the dielectric constant of the material. In these units, the effective Bohr radius is $a_{B}=1 / \beta$.

$\beta \rightarrow 0$ is a high-density (strong confinement) limit in which the Bohr radius is much higher than the oscillator length (equal to one in our units). The independent-electron and -hole picture works in this limit, and the Coulomb interaction may be computed in perturbation theory. Notice that at high density, we have a system of independent fermions, not 
bosons. This is one of the reasons preventing Bose condensation of excitons in homogeneous $3 \mathrm{D}$ systems [1].

On the other hand, as $\beta$ is increased, the dynamics become more and more dictated by the Coulomb forces. First, we shall observe the emergence of two-body correlations and the formation of electron-hole "Cooper" pairs (i. e. pairing in Fock space). With a further increase in $\beta$, small excitons, biexcitons and higher complexes shall start playing a dominant role.

Let us first consider the $\beta \rightarrow 0$ limit, in which the Coulomb interaction may be computed in perturbation theory. We will make an additional simplifying assumption: $N$ is such that there are $N_{\text {shell }}$ closed shells in the $\beta=0$ limit, that is the number of electrons takes one of the following values $N=N_{\text {shell }}\left(N_{\text {shell }}+1\right)=6,12,20,30,42, \ldots, 210$. The ground state of such systems for small $\beta$ values is spin-unpolarised, which means that both the electron and hole subsystems have total spin $S=0$. The angular momentum of this state is $L=0$.

The $\beta \rightarrow 0$ perturbative series take the form

$$
\frac{E}{\hbar \omega}=b_{0}+b_{1} \beta+b_{2} \beta^{2}+\mathcal{O}\left(\beta^{3}\right)
$$

where, the leading approximation to the energy is twice the energy of $N$ independent electrons (or holes)

$$
b_{0}=2 N \sqrt{4 N+1} / 3
$$

and for $b_{1}$ and $b_{2}$ we arrive to the following expressions

$$
\begin{gathered}
b_{1}=-2 \sum_{n_{1} \leq N / 2}\left\langle n_{1}, n_{1}|1 / r| n_{1}, n_{1}\right\rangle-4 \sum_{n_{1}<n_{2} \leq N / 2}\left\langle n_{1}, n_{2}|1 / r| n_{2}, n_{1}\right\rangle \\
b_{2}=-4 \sum_{n_{1} \leq N / 2} \sum_{n_{2}>N / 2} \frac{\left|\sum_{n \leq N / 2}\left\langle n_{2}, n|1 / r| n, n_{1}\right\rangle\right|^{2}}{\epsilon\left(n_{2}\right)-\epsilon\left(n_{1}\right)}-6 \sum_{n_{1} \leq N / 2} \sum_{n_{3}>N / 2} \frac{\left\langle n_{3}, n_{3}|1 / r| n_{1}, n_{1}\right\rangle^{2}}{\epsilon\left(n_{3}\right)-\epsilon(n 1)} \\
-24 \sum_{n_{1}<n_{2} \leq N / 2} \sum_{n_{3}>N / 2} \frac{\left\langle n_{3}, n_{3}|1 / r| n_{1}, n_{2}\right\rangle^{2}}{2 \epsilon\left(n_{3}\right)-\epsilon(n 1)-\epsilon\left(n_{2}\right)}-24 \sum_{n_{1} \leq N / 2} \sum_{n_{4}>n_{3}>N / 2} \frac{\left\langle n_{3}, n_{4}|1 / r| n_{1}, n_{1}\right\rangle^{2}}{\epsilon\left(n_{3}\right)+\epsilon(n 4)-2 \epsilon\left(n_{1}\right)} \\
-8 \sum_{n_{1}<n_{2} \leq N / 2} \sum_{n_{4}>n_{3}>N / 2}\left\{5\left\langle n_{3}, n_{4}|1 / r| n_{1}, n_{2}\right\rangle^{2}\right.
\end{gathered}
$$




$$
\begin{aligned}
& \left.+5\left\langle n_{3}, n_{4}|1 / r| n_{2}, n_{1}\right\rangle^{2}-4\left\langle n_{3}, n_{4}|1 / r| n_{1}, n_{2}\right\rangle\left\langle n_{3}, n_{4}|1 / r| n_{2}, n_{1}\right\rangle\right\} \\
& \times\left(\epsilon\left(n_{3}\right)+\epsilon\left(n_{4}\right)-\epsilon(n 1)-\epsilon\left(n_{2}\right)\right)^{-1} .
\end{aligned}
$$

The sums run over orbitals, which have been numbered sequentially. The sums over spin degrees of freedom have been explicitly evaluated. The Coulomb matrix elements are defined as

$$
\left\langle n_{1}, n_{2}|1 / r| n_{3}, n_{4}\right\rangle=\int \frac{\mathrm{d}^{2} r_{1} \mathrm{~d}^{2} r_{2}}{\left|\vec{r}_{1}-\vec{r}_{2}\right|} \phi_{n_{1}}^{*}\left(\vec{r}_{1}\right) \phi_{n_{2}}^{*}\left(\vec{r}_{2}\right) \phi_{n_{3}}\left(\vec{r}_{1}\right) \phi_{n_{4}}\left(\vec{r}_{2}\right) .
$$

The explicit form of the harmonic-oscillator orbitals is

$$
\phi_{k, l}=C_{k,|l|} r^{|l|} L_{k}^{|l|}\left(r^{2}\right) e^{-r^{2} / 2} e^{i l \theta}
$$

where $C_{k,|l|}=\sqrt{k ! /[\pi(k+|l|) !]}$, and $n=(k, l)$ is a composed index. The energy corresponding to $\phi_{k, l}$ is $\epsilon(k, l)=1+2 k+|l|$. In terms of these energies, we have $b_{0}=4 \sum_{n} \epsilon(n)$.

Numerical values for the coefficients $b_{1}$ and $b_{2}$ are presented in Table凹. The sums entering the $b_{2}$ coefficients were evaluated with a maximum of 20 shells. With respect to the number of shells included in the calculations, the convergence is slow, thus we used Shank extrapolants [18 to accelerate convergence. Fortunately, as a function of $N, b_{2}$ saturates very fast and there is no need to perform calculations for $N>42$. Notice the scaling laws $b_{0} \approx \frac{4}{3} N^{3 / 2}$, $b_{1} \approx-0.96 N^{5 / 4}, b_{2} \approx-1.65 N$ for $N \geq 42$. $b_{1}$ depends weaker on $N$ (as compared with electrons, for which the power is $7 / 4$ instead of $5 / 4$ [19]) because of the partial cancellation between attractive and repulsive Coulomb matrix elements.

Least-squares fits to the data in Table $\mathbb{\text { I lead to }}$

$$
\begin{aligned}
\frac{b_{1}}{N^{5 / 4}} & =-0.960853-\frac{0.355004}{N}+\frac{1.24679}{N^{2}}-\frac{3.37305}{N^{3}} \\
\frac{b_{2}}{N} & =-1.665+\frac{0.568}{N}-\frac{0.313}{N^{2}}
\end{aligned}
$$

\section{A. Approximate scaling and breakdown of the perturbative expansion}

For large enough $N$, we can use the asymptotic expressions for the coefficients to show that $E / N^{3 / 2}$ is approximately a function of the combination $N^{-1 / 4} \beta$. 


$$
\frac{E}{\hbar \omega N^{3 / 2}} \approx f\left(\beta / N^{1 / 4}\right) .
$$

The physics behind the scaling (10) is the following. As a result of cancellation between Coulomb attraction and repulsion, the size of the system is practically constant. Then, by increasing $N$, we increase the density and depress the effects of the Coulomb interaction. Approximate scaling of the energy is also characteristic of confined electron systems [19] and charged bosons in two dimensions [20]. Notice that in a pure electron system, where the interparticle potential is always repulsive, an increase in $N$ leads to a decrease of the density and an enhancement of correlation effects. The energy turns out to be a function of $N^{1 / 4} \beta$ at low $\beta$.

We shall stress that the scaling law (10) is expected to be observed also in the strong coupling, $\beta \rightarrow \infty$, limit in which the energy shall be roughly proportional to the energy of $N$ independent excitons,

$$
\left.\frac{E}{\hbar \omega}\right|_{\beta \rightarrow \infty}=a_{0} \beta^{2}+\cdots,
$$

where $a_{0} \approx-N$. The right hand side of Eq. (11) may thus be written as $N^{3 / 2}\left(-\beta^{2} / N^{1 / 2}\right)$. The variational results of the next sections also support the scaling behaviour (10).

A naive estimation of the convergence radius for the series (21) gives $\beta<\beta_{c}=b_{1} / b_{2}$. This estimation may be obtained formally as the pole of the Padé approximant

$$
P_{1,1}(\beta)=b_{0}+\frac{b_{1} \beta}{1+q_{1} \beta}, \quad q_{1}=-b_{2} / b_{1},
$$

which reproduces the expansion (2) for small $\beta$ values. Notice the high- $N$ asymptotic behaviour, $\beta_{c} \sim 0.58 N^{1 / 4}$. $\beta_{c}$ gives an estimate for the density at which exciton effects become important. Indeed, the density in our units is $\rho \approx N /\left(\pi\left\langle r^{2}\right\rangle\right) \approx 3 N^{1 / 2} /(2 \pi)$, thus $\beta_{c}$ may be expressed in terms of $\rho$. Turning back to ordinary units, we get a critical density, $\rho_{c} \sim 1 /\left(0.24 \pi a_{B}^{2}\right)$, i. e. approximately four times the close-packing density of excitons, $1 /\left(\pi a_{B}^{2}\right)$. This fact is consistent with the belief that screening is less effective in two dimensions. Below $\rho_{c}$, exciton effects shall dominate the quantum dynamics. As will be seen, $\rho_{c}$ is also at the onset of pairing in the BCS estimate of the next sections. 
In the following sections, we will perform variational estimations expected to be valid when pairing is not so strong, that is in the regime $\beta / N^{1 / 4} \leq 1$.

\section{VARIATIONAL ESTIMATIONS}

Let us turn to the variational calculations. The simplest variational estimation one can try is first-order perturbation theory.

$$
\frac{E}{\hbar \omega}<E_{P T 1}(\beta)=b_{0}+b_{1} \beta
$$

This estimate may be improved by introducing a frequency, $\Omega$, as an additional variational parameter, i.e. by taking as trial function the product of two Slater determinants of harmonic-oscillator states with a frequency $\Omega$. The result is,

$$
\frac{E}{\hbar \omega}<\min _{\Omega}\left\{\frac{1}{2}(\Omega+1 / \Omega) E_{P T 1}\left(\frac{2 \sqrt{\Omega}}{\Omega+1 / \Omega} \beta\right)\right\} .
$$

We checked that the result coming from (14) practically coincides with the Hartree-Fock (HF) energy for this system [11]. Thus, we will call (14) the HF estimate.

The mechanism by which the energy is lowered is pairing. We may take account of it with the help of a BCS-like wave function [21]. This may be a good estimation for weak pairing, when correlations are not so strong. In the $\beta$ axis, it means $\beta / N^{1 / 4}<1$. The wave function is given by

$$
|B C S\rangle=\prod_{j=1}^{N_{\max }}\left(u_{j}+v_{j} h_{j}^{+} e_{j^{\prime}}^{+}\right)|0\rangle_{h}|0\rangle_{e}
$$

$h_{j}^{+}$and $e_{j}^{+}$are hole and electron (harmonic oscillator) creation operators acting on their respective vacua $|0\rangle_{h}$ and $|0\rangle_{e} . j=\left(k, l, s_{z}\right)$ is a composed index, $j^{\prime}=\left(k,-l,-s_{z}\right)$. $s_{z}$ is the spin projection. $v_{j}$ and $u_{j}$ are normalised according to $u_{j}^{2}+v_{j}^{2}=1$. The total angular momentum corresponding to $|B C S\rangle$ is zero because the angular momentum of each pair is zero. The mean value of the total electron (hole) spin may be forced to be zero by requiring $v\left(k, l, s_{z}\right)=v\left(k, l,-s_{z}\right)$. Thus, $v_{j}$ does not depend on $s_{z}$ and we can write $v_{n}$ instead of $v_{j}$. 
$|B C S\rangle$ is not an eigenfunction of the particle number operator. In a finite system, we shall project onto the state with the correct number of particles. This will be done in two steps: first, an approximate projection before variation over the parameters $v_{n}$ entering the BCS function (the Lipkin-Nogami scheme [8]), and then an exact Monte Carlo projection of the BCS function onto the sector with $N$ pairs [9].

\section{A. The Lipkin-Nogami estimate}

In the Lipkin-Nogami (LN) method [8], one assumes an approximate polynomial dependence of $H$ on the particle number operator $\hat{N}$,

$$
H=\lambda_{0}+2 \lambda_{1} \hat{N}+\lambda_{2} \hat{N}^{2}
$$

By taking expectation values of $H$ over exact and BCS functions and comparing results, we arrive to

$$
E_{L N}=E_{B C S}-2 \lambda_{1}\left(\langle\hat{N}\rangle_{B C S}-N\right)-\lambda_{2}\left(\left\langle\hat{N}^{2}\right\rangle_{B C S}-N^{2}\right)
$$

where

$$
\begin{aligned}
E_{B C S}=\langle H\rangle_{B C S} & =\sum_{n}\left\{4 \epsilon_{n}-2 \beta\langle n, n|1 / r| n, n\rangle\right\} v_{n}^{2} \\
& -2 \beta \sum_{n_{1} \neq n_{2}}\left\langle n_{1}, n_{2}|1 / r| n_{2}, n_{1}\right\rangle\left\{v_{n_{1}}^{2} v_{n_{2}}^{2}+v_{n_{1}} u_{n_{1}} v_{n_{2}} u_{n_{2}}\right\} .
\end{aligned}
$$

Minimization over $\lambda_{1}$ leads to

$$
\begin{aligned}
N & =\left\langle\sum_{j} e_{j}^{+} e_{j}\right\rangle_{B C S}=\left\langle\sum_{j} h_{j}^{+} h_{j}\right\rangle_{B C S} \\
& =2 \sum_{n} v_{n}^{2} .
\end{aligned}
$$

The equation of minimum with respect to the $v_{n}$ can be written in the form of standard gap equations

$$
\Delta_{n}=\beta \sum_{n_{1} \neq n}\left\langle n, n_{1}|1 / r| n_{1}, n\right\rangle \frac{\Delta_{n_{1}}}{2 \sqrt{\Delta_{n_{1}}^{2}+\left(\epsilon_{n_{1}}^{H F}-\mu\right)^{2}}}
$$


where the HF energies are given by

$$
\epsilon_{n}^{H F}=\epsilon_{n}-\frac{\beta}{2}\langle n, n|1 / r| n, n\rangle-\beta \sum_{n_{1} \neq n}\left\langle n, n_{1}|1 / r| n_{1}, n\right\rangle v_{n_{1}}^{2}-\lambda_{2}\left(N-v_{n}^{2}\right),
$$

and we used the common BCS parametrization

$$
v_{n}^{2}=\frac{1}{2}\left(1-\frac{\epsilon_{n}^{H F}-\mu}{\sqrt{\Delta_{n}^{2}+\left(\epsilon_{n}^{H F}-\mu\right)^{2}}}\right) .
$$

The chemical potential, $\mu=\lambda_{1}+\lambda_{2} / 2$ was introduced in Eq. (22). For the determination of $\lambda_{2}$, the system of equations

$$
\begin{array}{r}
\left\langle H-\lambda_{0}-2 \lambda_{1} \hat{N}-\lambda_{2} \hat{N}^{2}\right\rangle_{B C S}=0, \\
\left\langle\left(H-\lambda_{0}-2 \lambda_{1} \hat{N}-\lambda_{2} \hat{N}^{2}\right) \hat{N}\right\rangle_{B C S}=0, \\
\left\langle\left(H-\lambda_{0}-2 \lambda_{1} \hat{N}-\lambda_{2} \hat{N}^{2}\right) \hat{N}^{2}\right\rangle_{B C S}=0,
\end{array}
$$

is used [8]. It makes the LN method not throughly variational. The first equation determines the constant $\lambda_{0}$. The second turns to be equivalent to the gap equation (20). For $\lambda_{2}$, we get

$$
\lambda_{2}=\frac{a_{1} a_{5}-a_{2} a_{4}}{a_{3} a_{5}-a_{2}^{2}}
$$

where

$$
\begin{gathered}
a_{1}=\left\langle H \hat{N}^{2}\right\rangle_{B C S}-\langle H\rangle_{B C S}\left\langle\hat{N}^{2}\right\rangle_{B C S}, \\
a_{2}=\left\langle\hat{N}^{3}\right\rangle_{B C S}-N\left\langle\hat{N}^{2}\right\rangle_{B C S} \\
a_{3}=\left\langle H \hat{N}^{4}\right\rangle_{B C S}-\left\langle\hat{N}^{2}\right\rangle_{B C S}^{2} \\
a_{4}=\langle H \hat{N}\rangle_{B C S}-N\langle H\rangle_{B C S} \\
a_{5}=\left\langle\hat{N}^{2}\right\rangle_{B C S}-N^{2}
\end{gathered}
$$

The resulting equations were solved iteratively starting from $\epsilon_{n}^{H F}=\epsilon_{n}, \Delta_{n}=0.2$. First, the explicit expressions for $v_{n}^{2}$ are used and the nonlinear equation (19) is solved for $\mu$. After 
that, we obtain $\lambda_{2}$ from (26), and the $\Delta_{n}$ and $\epsilon_{n}^{H F}$ are recalculated from (20,21). The process is repeated until the variation in any of the $\epsilon_{n}^{H F}$ is less than $10^{-10}$.

Calculations were carried out for $6 \leq N \leq 90$ pairs and a maximum of 600 one-particle states for both electrons and holes (i. e. 300 orbitals, because there are 2 spin states for each orbital). The absolute error in computing Coulomb matrix elements is less than $10^{-8}$. As is Eq. (14), we introduced an additional parameter $\Omega$, and used the inequality

$$
E \leq \min _{\Omega}\left\{\frac{1}{2}(\Omega+1 / \Omega) E_{L N}\left(\frac{2 \Omega^{1 / 2}}{\Omega+1 / \Omega} \beta\right)\right\},
$$

where $E_{L N}$ is the result from Eq. (17) at $\Omega=1$. The variation of $\Omega$ can be thought of as a simplified self-consistent Hartree-Fock-Bogoliubov procedure, in which the mean field is forced to be a harmonic potential.

We show in Fig. [ the energy coming from Eq. (32) versus $\beta$ for $N=6$ and 90 pairs (the curves $L N$ ). The HF estimates (14) and BCS curves (the $\lambda_{2}=0$ limit of LN) are also given for comparison. The lowest curves, labelled "proj", correspond to the exact projection of the next section. We notice that the LN method moves the BCS threshold for pairing towards zero, but the energy itself remains very close to the HF curve. A significant departure occurs only for $\beta>\beta_{c}$. $\left(\beta_{c} \approx 0.86\right.$ for $N=2$, and 1.7 for $\left.N=90\right)$.

\section{B. The Monte Carlo projection}

The next step is an exact projection of the wave function onto the $\mathrm{N}$-pair sector. The situation is similar to the calculations carried out for nuclei [22], where a variety of projection methods have been developed.

We project the wave function after the $\left\{v_{n}\right\}$ are determined for given $\beta$ and $\Omega$. With this function, the mean value of the hamiltonian at a shifted $\beta$ is computed and multiplied by the factor given in Eq. (32). The projected energy takes the following expression,

$$
\left.E\right|_{N}=\sum_{j_{1}, \ldots, j_{N}} W\left(j_{1}, \ldots, j_{N}\right) \varepsilon\left(j_{1}, \ldots, j_{N}\right),
$$


where the $j^{\prime}$ s have the same meaning as in Eq. (15), and the sum runs over possible combinations of $N$ states, $\left\{j_{1}, \ldots, j_{N}\right\}$, from a maximum of $N_{\text {max }}$ states allowed in the LN calculation. The "weights", $W$, and "energies", $\varepsilon$, are defined as

$$
\begin{gathered}
W\left(j_{1}, \ldots, j_{N}\right)=\frac{v_{j_{1}}^{2} \ldots v_{j_{N}}^{2}}{u_{j_{1}}^{2} \ldots u_{j_{N}}^{2}}\left(\sum_{j_{1}^{\prime}, \ldots, j_{N}^{\prime}} \frac{v_{j_{1}^{\prime}}^{2} \ldots v_{j_{N}^{\prime}}^{2}}{u_{j_{1}^{\prime}}^{2} \ldots u_{j_{N}^{\prime}}^{2}}\right)^{-1}, \\
\varepsilon\left(j_{1}, \ldots, j_{N}\right)=2 \sum_{j \in\left\{j_{1}, \ldots, j_{N}\right\}} \epsilon_{j}-\beta \sum_{j \in\left\{j_{1}, \ldots, j_{N}\right\}}\langle j, j|1 / r| j, j\rangle-\beta \sum_{j, j^{\prime} \in\left\{j_{1}, \ldots, j_{N}\right\}, j \neq j^{\prime}}\left\langle j, j^{\prime}|1 / r| j^{\prime}, j\right\rangle \\
-\beta \sum_{j \in\left\{j_{1}, \ldots, j_{N}\right\}, j^{\prime} \notin\left\{j_{1}, \ldots, j_{N}\right\}}\left\langle j, j^{\prime}|1 / r| j^{\prime}, j\right\rangle \frac{u_{j} v_{j^{\prime}}}{u_{j^{\prime}} v_{j}}
\end{gathered}
$$

The expression (33) for the projected energy allows a simple Monte Carlo evaluation, where the sets $\left\{j_{1}, \ldots, j_{N}\right\}$ are generated with probability $W\left(j_{1}, \ldots, j_{N}\right)$ by means of a Metropolis algorithm [23]. Other equivalent forms of Eq. (33), see for example Ref. [24], are not suited for this evaluation. The procedure seems to be particularly efficient in Nuclear Physics calculations as well [9].

The results are also drawn in Fig. 1. The improvement is significant for $\beta \sim \beta_{c}$, and its relative importance diminishes as $N$ is increased.

\section{Results}

We show in Fig. 2 our best results for the energies of the systems under study. The scaled energies show a remarkable similarity. A significant pairing (i. e. departure from the HF curve) is seen only for $\beta / N^{1 / 4} \geq 0.55$.

Finally, we give a parametrisation of the ground-state energy obtained from the best of our variational estimates. The energy is written in the form of a Padé approximant [25],

$$
E_{g s}=b_{0}+b_{1} \beta+\frac{b_{2} \beta^{2}+p_{3} \beta^{3}+p_{4} \beta^{4}}{1+q_{1} \beta+q_{2} \beta^{2}}
$$

where $p_{3}=q 1 p 4 / q 2-b 1 q 2$, and the coefficients $p_{4}, q_{1}$, and $q_{2}$ are fitted from our numerical

results. The obtained values are shown in Table @. 


\section{CONCLUDING REMARKS}

We have studied electron-hole systems in quantum dots under strong and intermediate confinement, where the dense electron-hole or the BCS excitonic phases are present.

The breakdown of perturbation theory and a significant pairing in the BCS wave function, both take place at a density which is roughly four times the close-packing density of excitons. We interpret this result as a crossover between the two phases.

As mentioned before, with an increase in $\beta$, particle correlations shall become more and more important. We shall observe signals of the "excitonic", "biexcitonic", etc. insulating phases. The true energies and wave functions of these phases shall be obtained with

more powerful methods as, for example, Green-function Monte Carlo method [23]. Even a variational Monte Carlo estimation, as that one carried out for the homogeneous case in Ref. [26], may be biased by the chosen trial functions. The density matrix renormalisation group method of Ref. [27] could also be useful. The very interesting question about whether the system remains bound after the external potential is switched off, still remains to be answered. We have some indications that the two-dimensional triexciton is bound and the four-exciton system is unbound [12]. But the situation may be analogous to nuclei, where there is a small instability island around atomic number 5. Some of these problems are currently under investigation.

\section{ACKNOWLEDGMENTS}

The authors acknowledge support from the Colombian Institute for Science and Technology (COLCIENCIAS). Part of this work was done during a visit of A. G. and B. R. to the Abdus Salam ICTP under the Associateship Scheme and the Visiting Young Student Programme. 


\section{REFERENCES}

a E-mail: brodrigz@democritus.udea.edu.co

b E-mail: agonzale@democritus.udea.edu.co

c E-mail: lquiroga@uniandes.edu.co

d E-mail: rcapote@infomed.sld.cu

e E-mail: ferney@anacaona.uniandes.edu.co

[1] See, for example, L. V. Keldysh in Bose-Einstein condensation, eds. A. Griffin, D. W. Snoke and S. Stringari, Cambridge Univ. Press, 1995, pag. 246, and references therein.

[2] M. H. Anderson, J. R. Ensher, M. R. Mathews et al, Science 269, 198 (1995); K. B. Davis, M. O. Mewes, M. R. Andrews et al, Phys. Rev. Lett. 75, 3969 (1995); C. C. Bradley, C. A. Sackett, J. J. Tollet and R. G. Hulet, Phys. Rev. Lett. 75, 1687 (1995).

[3] J. C. Kim and J. P. Wolfe, Phys. Rev. B 57, 9861 (1998).

[4] V. Negoita, D. W. Snoke and K. Eberl, Phys. Rev B 60, 2661 (1999).

[5] G. Bastard and B. Gil (eds.), Optics of excitons in confined systems, Journal de Physique IV, Vol. 3, Colloque C 3 (1993).

[6] R. Ambigapathy, I. Bar-Joseph, D. Y. Oberli et al, Phys. Rev. Lett. 78, 3579 (1997).

[7] E. Dekel, D. Gershoni, E. Ehrenfreund, D. Spektor, J. M. Garcia and P. M. Petroff, Phys. Rev. Lett. 80, 4991 (1998); E. Dekel, D. Gershoni, E. Ehrenfreund, J. M. Garcia and P. M. Petroff, cond-mat/9904334.

[8] H. C. Pradham, Y. Nogami and J. Law, Nucl. Phys. A 201, 357 (1973); J. Dobaczewski and W. Nozarewickz, Phys. Rev. C 47, 2418 (1993).

[9] R. Capote and A. Gonzalez, Phys. Rev. C 59, 3477 (1999).

[10] M. Rho and J. O. Rasmussen, Phys. Rev. 135, B1295 (1964). 
[11] A. Gonzalez, R. Capote, A. Delgado and L. Lavin, cond-mat/9809399, submitted.

[12] R. Perez and A. Gonzalez, submitted.

[13] J. Speth (ed.), Electric and magnetic giant resonances in nuclei, World Scientific, Singapore (1991).

[14] J. P. Connerade (ed.), Correlations in clusters and related systems, World Scientific, Singapore (1996).

[15] A. de Shalit and H. Feschbach, Theoretical Nuclear Physics, Vol. I, John Wiley \& Sons, New York (1974).

[16] R. Coté and A. Griffin, Phys. Rev. B 37, 4539 (1988).

[17] A. Wojs, P. Hawrylak, S. Fafard, and L. Jacak, Phys. Rev. B 54, 5604 (1996).

[18] C. M. Bender and S. A. Orszag, Advanced mathematical methods for scientists and engineers, McGraw-Hill, New-York, 1978.

[19] A. Gonzalez, B. Partoens and F. M. Peeters, Phys. Rev. B 56, 15740 (1997).

[20] A. Gonzalez, B. Partoens, A. Matulis and F. M. Peeters, Phys. Rev. B 59, 1653 (1999).

[21] C. P. Enz, A course on many-body theory applied to Solid State Physics, World Scientific, 1992.

[22] D. C. Zheng, D. W. L. Sprung and H. Flocard, Phys. Rev. C 46, 1355 (1992).

[23] D. M. Ceperley, in Spring College in Computational Physics, ICTP, Trieste, 1997.

[24] V. G. Soloviev, Theory of complex nuclei, New-York, Oxford, 1976.

[25] L. Quiroga, F. J. Rodriguez and A. Gonzalez, Proceedings of ICPS-24, Jerusalem, 1998, in press.

[26] X. Zhu, M. S. Hybertsen and P. B. Littlewood, Phys. Rev. B 54, 13575 (1996). 
[27] J. Dukelsky and G. Sierra, Phys. Rev. Lett. 83, 172 (1999). 


\section{FIGURES}

FIG. 1. a) and b): Ground-state energies of the 6-exciton and 90-exciton systems respectively.

FIG. 2. Scaling of the ground-state energies 


\section{TABLES}

TABLE I. The coefficients $b_{1}, b_{2}, p_{3}, p_{4}, q_{1}$, and $q_{2}$.

\begin{tabular}{|c|c|c|c|c|c|c|}
\hline$N$ & $b_{1} / N^{5 / 4}$ & $b_{2} / N$ & $p_{3}$ & $p_{4}$ & $q_{1}$ & $q_{2}$ \\
\hline 6 & -1.001 & -1.58 & 21.9817 & -461906 & 15.3098 & 9.92132 \\
\hline 12 & -0.983778 & -1.62 & 43.4125 & -47.3284 & 6.46992 & 4.84953 \\
\hline 20 & -0.9758 & -1.64 & 53.9705 & -47.5022 & 3.60744 & 2.85761 \\
\hline 30 & -0.971391 & -1.65 & 84.1615 & -64.3801 & 3.68693 & 2.58195 \\
\hline 42 & -0.968681 & -1.65 & 127.946 & -115.014 & 6.85897 & 3.44577 \\
\hline 56 & -0.966881 & & 172.825 & -157.173 & 8.02801 & 3.55984 \\
\hline 72 & -0.965621 & & 213.377 & -189.426 & 8.25862 & 3.35560 \\
\hline 90 & -0.9647 & & 259.185 & -224.076 & 8.27331 & 3.16176 \\
\hline 110 & -0.964009 & & & & & \\
\hline 132 & -0.963475 & & & & & \\
\hline 156 & -0.963055 & & & & & \\
\hline 182 & -0.962712 & & & & & \\
\hline 210 & -0.962433 & & & & & \\
\hline
\end{tabular}




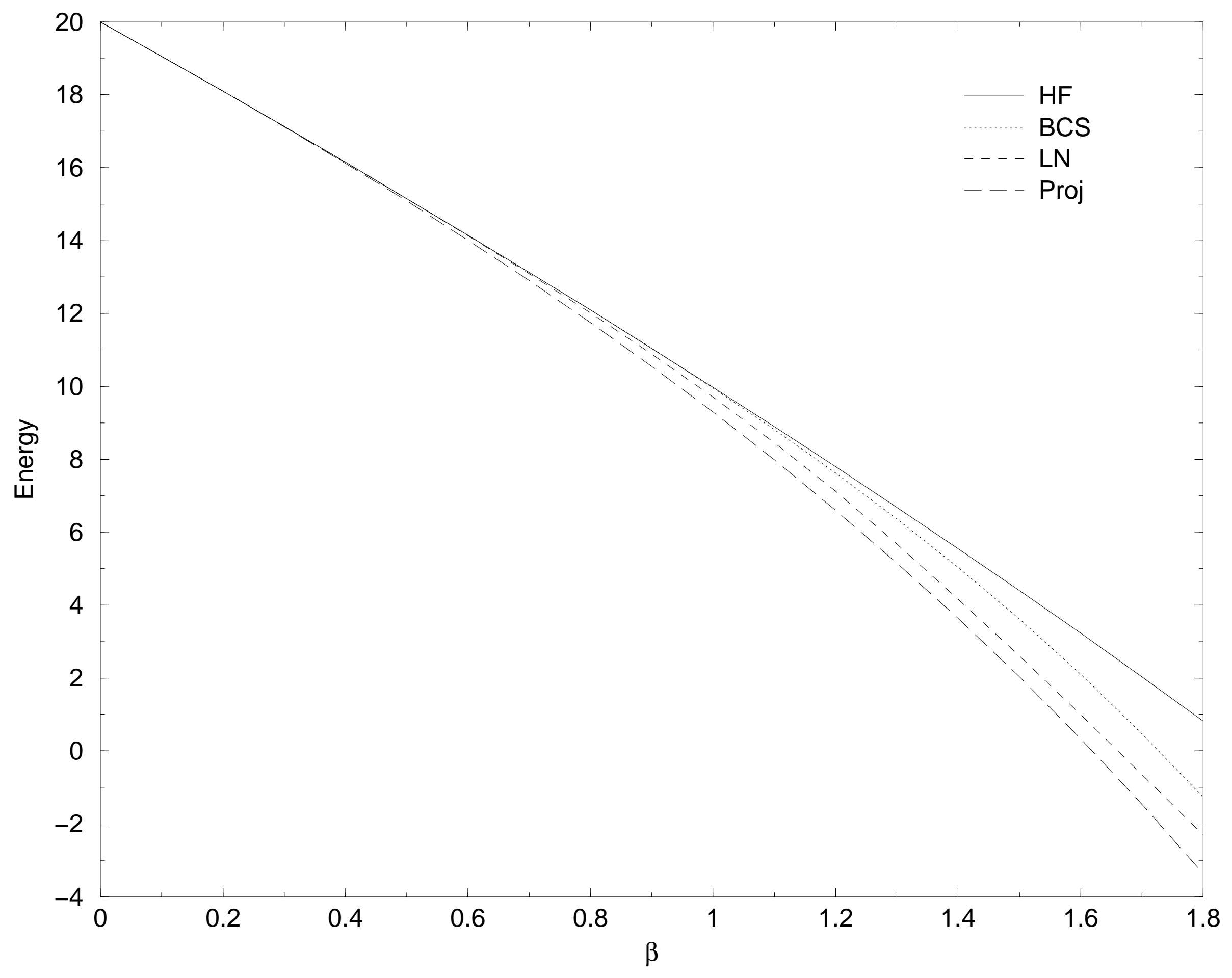




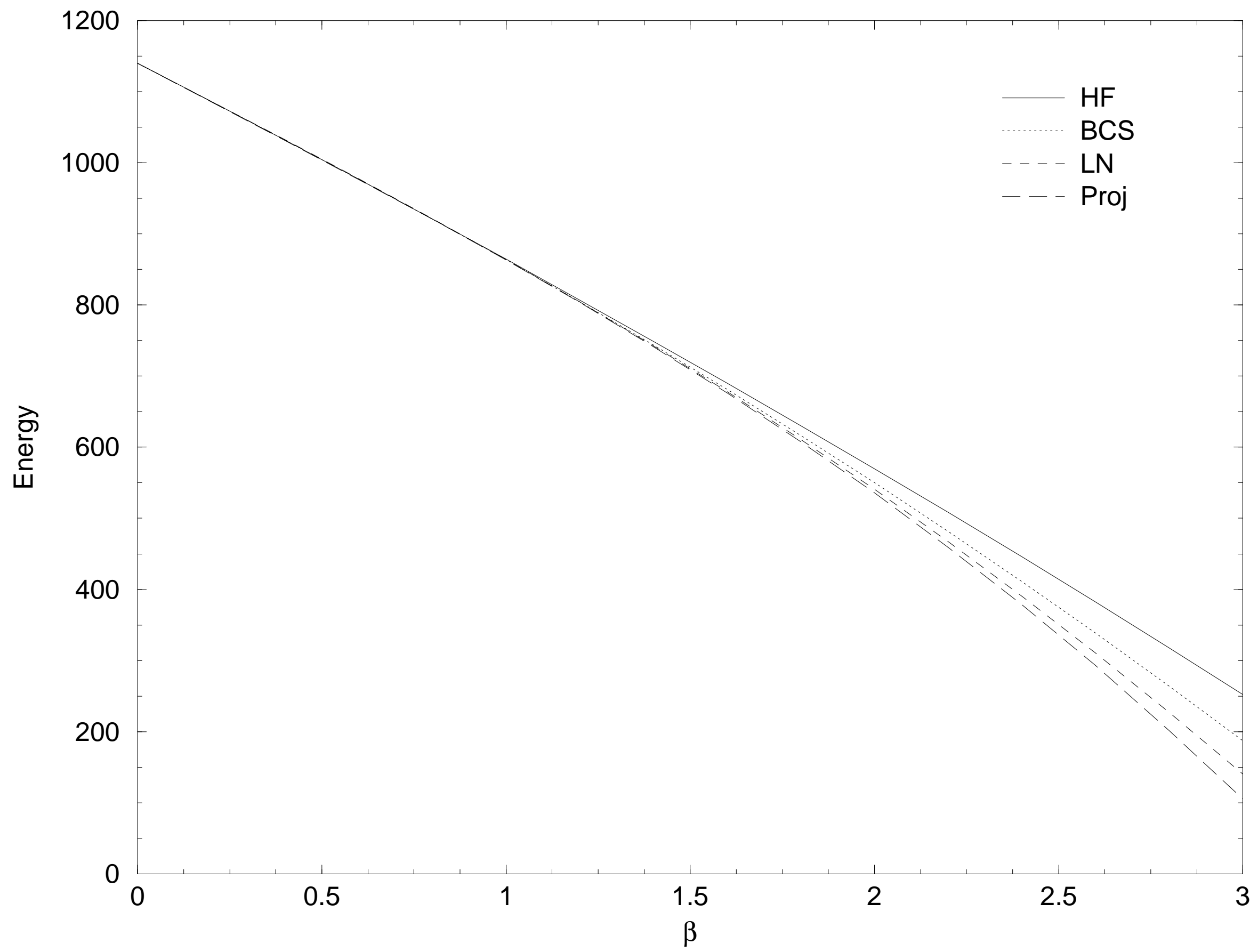




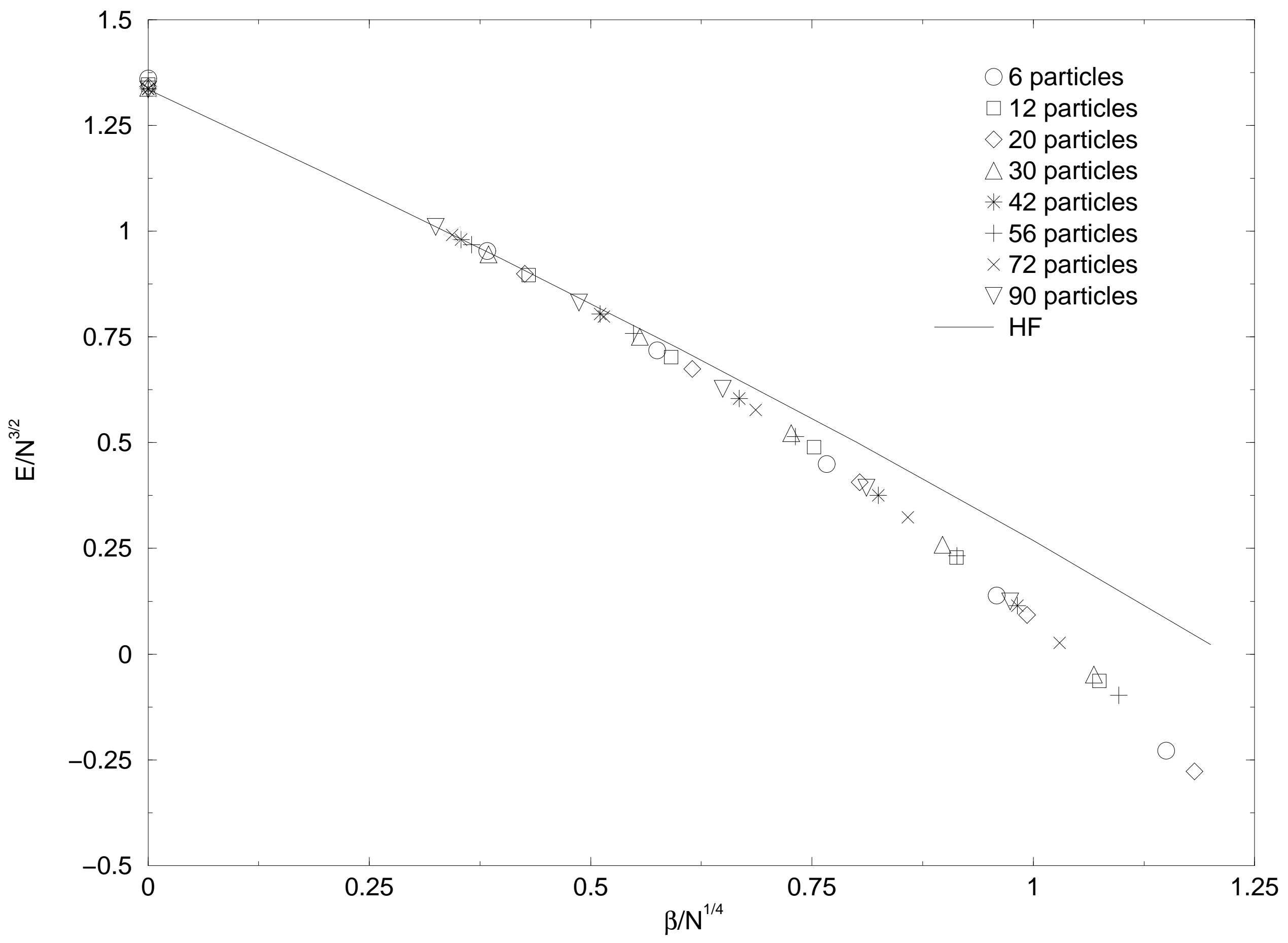

